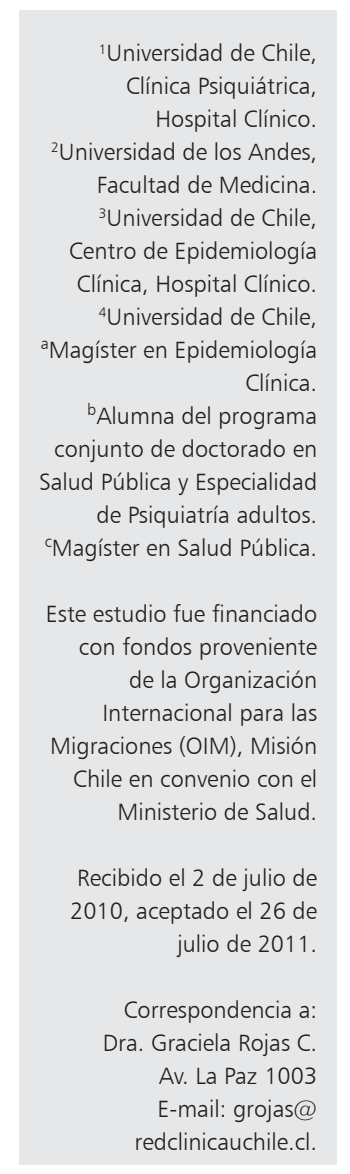

\section{Trastornos mentales comunes y uso de servicios de salud en población inmigrante}

\author{
GRACIELA ROJAS $^{1}$, ROSEMARIE FRITSCH ${ }^{1,2}$, ARIEL CASTRO $^{3, a}$, \\ VIVIANA GUAJARDO ${ }^{1,4, b}$, PAMELA TORRES ${ }^{4, c}$, BERTA DÍAZ $^{1}$
}

\section{Mental disorders among immigrants in Chile}

Background: Chile is receiving immigrant populations coming from other LatinAmerican countries. Aim: To determine the prevalence of Common Mental Disorders (CMD) among immigrants who live in Independencia, a quarter in Santiago, Chile. Material and Methods: A cross sectional study was carried out in the primary health care clinic and in the state-funded school of Independencia. A representative sample of 282 adults and 341 children were interviewed. Mental disorders were diagnosed using CIS-R and MINI structured interviews. Results: The interviewed immigrants came mostly from Peru. The prevalence of mental disorders in the adult population was $17.8 \%$ and among children, it was $29.3 \%$. Conclusions: The adult immigrants have a lower prevalence of mental disorders than the Chilean population but it increases among children. Barriers of access to health services, that should be solved, were detected.

(Rev Med Chile 2011; 139: 1298-1304).

Key words: Chile; Emigrants and inmigrants; Mental health; Perú.
L

a migración se define como el "desplazamiento geográfico de individuos o grupos, generalmente por causas económicas o sociales" (Diccionario de la Real Academia Española).

El fenómeno migratorio ha existido en las diversas sociedades. En las últimas décadas, el avance tecnológico y de las comunicaciones ha originado movimientos migratorios relevantes ${ }^{1}$.

Al año 2000, se calcula en 175 millones el número de inmigrantes a nivel mundial, lo que corresponde a $3 \%$ de la población ${ }^{2}$. En Chile, asciende a 184.464 personas, lo que corresponde a $1,2 \%$ de la población nacional (INE) 3 .

Históricamente, Chile fue receptor de inmigrantes provenientes de países de Europa, Asia y África. Sin embargo, en la actualidad la mayor cantidad de inmigrantes proviene de países latinoamericanos, principalmente Argentina 38\% y Perú $30 \%{ }^{3}$.
En la última década, se ha asentado en la comuna de Independencia una población inmigrante que asciende a 2,4\%, el doble que la del país.

La inmigración es considerado un evento vital que requiere que el individuo despliegue al máximo sus capacidades para lograr una adecuada adaptación a su nuevo entorno, lo que pudiese afectar su salud.

$\mathrm{Al}$ respecto, se han realizado estudios que muestran que la autopercepción de salud general y salud mental de este grupo sería de regular a mala ${ }^{4,5}$. Además, se ha evidenciado que la salud mental en los inmigrantes puede estar afectada negativamente por el mismo proceso migratorio ${ }^{6,7}$.

Estudios realizados en Estados Unidos de Norteamérica, han reportado que la tasa de trastornos psiquiátricos de la población inmigrante es menor en comparación a los sujetos nacidos en ese país, ${ }^{4,8}$. En Europa, las cifras encontradas son variadas. En España, Bélgica y Alemania se encontró una mayor 
prevalencia de trastornos depresivos, de ansiedad y somatización en grupos inmigrantes, en comparación con la población nativa ${ }^{9-11}$. En Suiza, los inmigrantes provenientes de países de la ex-Unión Soviética, presentan tasas similares de sintomatología psiquiátrica comparado con los nacidos en Suiza, no así los inmigrantes provenientes de otros países del Este de Europa que presentan el doble de sintomatología psiquiátrica ${ }^{5}$.

En niños y adolescentes inmigrantes las tasas de prevalencia de depresión, trastornos de ansiedad y del comportamiento son similares a las de los niños nacidos en Holanda y en Austria ${ }^{12,13}$.

Se han descrito barreras financieras y culturales en el acceso de los inmigrantes a los sistemas de salud, falta de confianza en ellos y carencia de información sobre los derechos ${ }^{14-17}$.

En Chile, no existe a la fecha conocimiento respecto a la salud mental de la población inmigrante la que es necesaria para la definición de políticas de salud hacia esta población.

El objetivo de este estudio es determinar la prevalencia de sintomatología y patología psiquiátrica de la población inmigrante que reside en la comuna de Independencia y las barreras de acceso a la atención por problemas de salud mental.

\section{Método}

Se llevó a cabo un estudio de tipo epidemiológico transversal en 2 poblaciones, una adulta y otra infantojuvenil (IJ) entre de agosto del año 2007 y marzo del año 2008.

Al no contar con un marco muestral para la población inmigrante residente en la comuna de Independencia, se construyeron dos marcos muestrales: uno con la población adulta inmigrante del Consultorio Cruz Melo de la comuna de Independencia -a esa fecha el único de la comuna con población inmigrante- y una IJ con los escolares extranjeros matriculados en establecimientos educacionales municipales y particular subvencionadas de la misma comuna

Para el marco muestral de los adultos, se consideraron los registros del consultorio que consideraban 1.030 pacientes extranjeros adultos de 15 a 64 años. Con una prevalencia de patología psiquiátrica en la atención primaria en Chile cercana al $50 \%{ }^{18}$ y un error alfa de 0,05 , el tamaño muestral fue de 281 usuarios.
Para el marco muestral IJ, se procedió a censar a toda la población escolar de colegios municipalizados y subvencionados de la comuna de Independencia. De un total de 46 establecimientos educacionales registrados, se seleccionaron los que tenían alumnos extranjeros entre los 6 y los 16 años de edad capaces de responder cuestionarios, con residencia en la comuna de Independencia. De ellos, 32 cumplían dichos criterios (9 Municipales y 23 Particulares Subvencionados), los cuales fueron contactados solicitando información acerca del número de niños extranjeros matriculados a la fecha en su establecimiento educacional, dando un resultado de 878 alumnos que constituyeron el universo del estudio. Se calculó que una muestra aleatoria de 341 niños, es representativa de este universo, con una prevalencia cercana al 50\% de patología psiquiátrica ${ }^{18}$, una pérdida del $20 \%$ y un error alfa de 0,05 , con una prevalencia esperada del parámetro a evaluar, más desfavorable $(\mathrm{p}=0,5)$.

\section{Instrumentos}

\section{Población adulta}

Previo consentimiento informado aprobado por el comité de Ética del Hospital Clínico de la Universidad de Chile, cada individuo fue entrevistado con un instrumento elaborado basado en el cuestionario del estudio "Salud Mental en el Gran Santiago", que incluía: un instrumento de tamizaje (GHQ) ${ }^{19}$ una sección sociodemográfica, una sección acerca de la trayectoria migratoria y otra sobre uso de servicios de salud y satisfacción usuaria $^{20}$. La patología psiquiátrica fue evaluada con la Entrevista Clínica Estructurada (CIS-R), validada y utilizada en estudios en la atención primaria en Chile ${ }^{20}$. El CIS-R consta de 14 secciones que cubren los síntomas neuróticos más importantes. La presencia de TMC fue definida como un puntaje total del CIS-R de 12 o más puntos. Para medir específicamente, Trastorno por Estrés Postraumático, se usó la sección respectiva del MINI que es una entrevista que explora en forma estandarizada los criterios diagnósticos del DSMIV, ha sido validada en español y ampliamente utilizada en Chile.

\section{Población IJ inmigrante escolarizada}

Previo consentimiento informado de los padres y asentimiento de los niños aprobado por el 
mismo comité que los adultos, cada escolar fue evaluado mediante instrumentos autoaplicados, que incluían: el Cuestionario Pediátrico de Síntomas (PSC) y el MINI para niños y adolescentes.

El PSC desarrollado por Jellinek y cols es contestado por los padres de los escolares. Tiene 35 ítems, que se puntúan de 1 a 3 . Entrega un puntaje global que refleja la visión del padre acerca del funcionamiento psicosocial del escolar. Con un punto de corte mayor o igual a 28 puntos, indica que el escolar pudiese requerir atención en salud mental ${ }^{21}$. Adaptado y validado en escolares de enseñanza básica en Chile por George y $\operatorname{cols}^{22}$.

Además se administraron cuestionarios a los apoderados para evaluar presencia de trastornos emocionales en los escolares, datos sociodemográficos y del proceso migratorio.

\section{Análisis de los datos}

Se aplicó estadística descriptiva, según el tipo de variable y se aplicó la prueba de chi cuadrado para el análisis de las variables cuantitativas. Se consideró una significancia de $\mathrm{p}<0,05$.

\section{Resultados}

\section{Descripción de la muestra adulta}

Se seleccionó una muestra consecutiva de 282 pacientes inmigrantes adultos entre 15 y 62 años, los cuales fueron contactados en la sala de espera del consultorio, 222 eran mujeres (78,7\%). La mediana de la edad de fue 29 años. La mayoría de los consultantes convivía con una pareja $(51,4 \%), 23 \%$ era casado(a), 22,3\% soltero(a), $2,8 \%$ separado(a) y $0,3 \%$ era viudo(a). $64,9 \%$ había cursado enseñanza media, 20,2\% superior y $14,9 \%$ básica.

La gran mayoría de los entrevistados provenía de Perú (96,8\%). El promedio de estadía en Chile era de 51 meses, es decir, entre 4 y 5 años; con una estadía máxima de 16 años en Chile.

El $64,5 \%$ pertenecían a FONASA y $34,4 \%$ de los consultantes no tenían previsión (Tabla 1).

\section{Descripción de la muestra escolar}

Se evaluaron 341 niños entre 6 y 16 años en sus respectivos establecimientos educacionales. El 52,7\% de la muestra eran hombres. La edad promedio fue de 12 años. La gran mayoría (92\%) de los escolares provenía de Perú, en menor proporción de Ecuador y Argentina.
El promedio de residencia en Chile de los escolares era de 3 a 4 años, con un rango entre menos de un año hasta 14 años. El 81,3\% residía en el país desde hace menos de 5 años.

Los alumnos entrevistados cursaban de $1^{\circ}$ básico a cuarto medio, concentrándose mayoritariamente desde quinto básico a segundo medio (Tabla 2).

\section{Salud mental y barreras de acceso a los servicios de salud de la población adulta}

El 48,9\% de los consultantes adultos al Consultorio Cruz Melo calificó su salud mental como "buena". (Tabla 3) No se encontraron diferencias significativas entre los sexos en la auto-percepción de la salud mental.

La población adulta declaró que cuando ha tenido problemas de salud principalmente ha recurrido a la atención primaria y ha quedado satisfecha con la atención recibida. Hay $26 \%$ de

Tabla 1. Caracterización sociodemográfica de la población adulta consultante

\begin{tabular}{|lc|}
\hline & $N=282$ \\
\hline Sexo & Mujeres $222(78,7 \%)$ \\
& Hombres $60(21,3 \%)$ \\
\hline Edad (años) & $29 \pm(15-62)$ \\
\hline Estado Civil & \\
Casado & $65(23,0 \%)$ \\
Conviviente & $145(51,4 \%)$ \\
Viudo(a) & $1(0,3 \%)$ \\
Separado(a) & $8(2,8 \%)$ \\
Soltero(a) & $63(22,3 \%)$ \\
Educación & \\
Básica & $42(14,9 \%)$ \\
Media & $183(64,9 \%)$ \\
Superior & $57(20,2 \%)$ \\
País origen & \\
Ecuador & $8(2,8 \%)$ \\
Perú & $273(96,8 \%)$ \\
Venezuela & $1(0,3 \%)$ \\
Previsión de Salud & \\
FONASA & $182(64,54 \%)$ \\
ISAPRE & $1(0,3 \%)$ \\
No tiene & $97(34,4 \%)$ \\
No sabe & $2(0,7 \%)$ \\
Años de residencia en Chile & \\
1 - 5 años & $196(69,5 \%)$ \\
6 - 10 años & $74(26,24 \%)$ \\
$11-16$ años & $12(4,25 \%)$ \\
\hline
\end{tabular}


Trastornos mentales en población inmigrante - G. Rojas et al

Tabla 2. Caracterización sociodemográfica de la población escolar

\begin{tabular}{|lc|}
\hline \multicolumn{1}{|c|}{$N=300$} \\
\hline Sexo & $\begin{array}{c}\text { Mujeres 142 (47,3\%) } \\
\text { Hombres 158 (52,7\%) }\end{array}$ \\
\hline Edad (años) & $12 \pm 2,9(6-16)$ \\
\hline País origen & \\
Ecuador & $15(5 \%)$ \\
Perú & $276(92 \%)$ \\
Argentina & $3(1 \%)$ \\
Otro & $6(2 \%)$ \\
Educación & \\
Básica & $213(71 \%)$ \\
Media & $87(29 \%)$ \\
Años de residencia en Chile & \\
$0-5$ años & $234(81,3 \%)$ \\
6-10 años & $50(17,4 \%)$ \\
$11-14$ años & $4(1,4 \%)$ \\
Años de residencia en Chile & $3,4 \pm 2,6(0-14)$ \\
\hline
\end{tabular}

personas, que no ha consultado cuando ha tenido problemas de salud, ya sea por "desconocimiento del sistema de salud chileno" (72,7\%), por el costo $(63,6 \%)$ o por no tener rol único tributario (RUT) $(63,6 \%)$, entre otras razones (Tabla 4$)$.

\section{Prevalencia de patología psiquiátrica población adulta}

El 14,2\% de los entrevistados tenía algún trastorno psiquiátrico, según el CIS-R, a los que se debe agregar $3,6 \%$ que presenta sólo estrés postraumático, es decir, la prevalencia de TMC es $17,8 \%$. El síntoma que presentaron con mayor frecuencia las personas entrevistadas fue fatiga $(13,8 \%)$, seguido de ideas depresivas, preocupaciones, depresión e irritabilidad. Algunos síntomas se encontraron significativamente más presentes en las mujeres que en los hombres: : los síntomas somáticos $\left(\mathrm{M}=10,8 \%-\mathrm{H}=0 \% ; \chi^{2}=7,090\right.$; $\mathrm{p}=0,002)$, la fatiga $(\mathrm{M}=17,1 \%-\mathrm{H}=1,7 \%$; $\left.\chi^{2}=9,462 ; \mathrm{p}=0,001\right)$, los problemas de concentración $\left(\mathrm{M}=10,8 \%-\mathrm{H}=0 \% ; \chi^{2}=7,090 ; \mathrm{p}=0,002\right)$, la irritabilidad $\left(\mathrm{M}=12,2 \%-\mathrm{H}=3,3 \% ; \chi^{2}=3,991\right.$; $\mathrm{p}=0,0031)$ y la depresión $(\mathrm{M}=12,6 \%-\mathrm{H}=1,7 \%$; $\left.\chi^{2}=6,134 ; \mathrm{p}=0,006\right)$.

El cuadro psiquiátrico más prevalente fue el trastorno ansioso-depresivo (6,0\%), seguido por la depresión severa $(3,2 \%)$, que alcanza cifras
Tabla 3. Percepción de salud mental de la población adulta consultante

\begin{tabular}{|lrllrr|}
\hline $\begin{array}{l}\text { Salud } \\
\text { general }\end{array}$ & n & $\mathbf{( \% )}$ & $\begin{array}{l}\text { Salud } \\
\text { mental }\end{array}$ & n & (\%) \\
Muy buena & 17 & $(6)$ & Muy buena & 9 & $(3,2)$ \\
Buena & 139 & $(49,3)$ & Buena & 138 & $(48,9)$ \\
Regular & 112 & $(39,7)$ & Regular & 128 & $(45,4)$ \\
Mala & 12 & $(4,3)$ & Mala & 6 & $(2,1)$ \\
Muy mala & 2 & $(0,7)$ & Muy mala & 1 & $(0,4)$ \\
Problema & & & & & \\
salud & & & & & \\
Sí & $65(23)$ & & & \\
\hline No & $217(77)$ & & & \\
\hline
\end{tabular}

Tabla 4. Razones para no consultar a pesar de haber tenido problemas de salud

\begin{tabular}{|lc|}
\hline Razones & n (\%) \\
\hline Problemas se resolverían solos & $12(54,5)$ \\
\hline Preocupación por costo & $14(63,6)$ \\
\hline Sin RUT & $14(63,6)$ \\
\hline Desconocimiento Sistema Salud Chileno & $16(72,7)$ \\
\hline Sin permiso laboral & $11(50,0)$ \\
\hline Desconfianza Sistema Salud Chileno & $9(40,9)$ \\
\hline
\end{tabular}

similares al trastorno por estrés postraumático $(3,6 \%)$. Estas 3 patologías tienen una prevalencia en conjunto de $12,8 \%$ (Tabla 5). No se encontraron diferencias estadísticamente significativas entre los sexos en la prevalencia de estos cuadros psiquiátricos.

Percepción de salud mental y barreras de acceso a los servicios de salud de la población escolar

Treinta y dos coma cuatro porciento de los escolares entrevistados percibió tener un problema emocional o de comportamiento por el cual necesitaba ayuda, 9,6\% de los escolares declaró estar actualmente consultando a un profesional de la salud mental (Tabla 6). El 36,1\% de los apoderados perciben que su pupilo /a tiene un problema de salud mental. Las razones más frecuentes de no consultar a pesar de percibir un problema de salud mental según el apoderado, serían preocupacio- 
Tabla 5. Problemas de salud mental de la población adulta consultante

\begin{tabular}{|lrc|}
\hline & n & $(\%)$ \\
Presencia de trastorno psiquiátrico & 50 & $(17,8)$ \\
Síntomas psiquiátricos & & \\
Fatiga & 39 & $(13,8)$ \\
Ideas depresivas & 32 & $(11,3)$ \\
Preocupación & 31 & $(11)$ \\
Depresión & 29 & $(10,3)$ \\
Irritabilidad & 29 & $(10,3)$ \\
Síntomas somáticos & 24 & $(8,5)$ \\
Problemas de concentración & 24 & $(8,5)$ \\
Preocupaciones por salud física & 19 & $(6,7)$ \\
Pánico & 12 & $(4,3)$ \\
Ansiedad & 10 & $(3,5)$ \\
Problemas de sueño & 7 & $(2,5)$ \\
Fobias & 7 & $(2,5)$ \\
Trastorno ansioso-depresivo & 17 & $(6,0)$ \\
Depresión & & \\
Severa & 9 & $(3,2)$ \\
Moderada & 3 & $(1,1)$ \\
Leve & 2 & $(0,7)$ \\
Trastorno de pánico & 4 & $(1,4)$ \\
Trastorno obsesivo compulsivo & 4 & $(1,4)$ \\
Agorafobia & 1 & $(0,4)$ \\
Trastorno por estrés postraumático & 10 & $(3,6)$ \\
\hline & & \\
\hline
\end{tabular}

nes por el costo $(64,4 \%)$, pensar que los problemas se resolverían solos $(56,7 \%)$ o desconocimiento del sistema de salud chileno $(48,9 \%)$ (Tabla 7$)$.

\section{Prevalencia de patología psiquiátrica población escolar}

El $29,3 \%$ de la población escolar entrevistada percibe un malestar emocional significativo. Se trata de trastornos mentales comunes: $14,3 \%$ trastornos del ánimo y 5,7\% trastornos de ansiedad. Las mujeres presentaron significativamente más trastornos de ánimo (Tabla 8).

\section{Discusión}

La prevalencia de 17,8\% de trastornos mentales comunes entre la población inmigrante adulta estudiada es inferior a las cifras encontradas en las investigaciones llevadas a cabo en nuestro país en consultantes a la atención primaria, que es cercana a $50 \%{ }^{23}$.
Tabla 6. Percepción de salud de la población escolar y apoderado.

\begin{tabular}{|lc|}
\hline & $\begin{array}{c}\text { Según el } \\
\text { alumno } \\
\mathbf{n}(\%)\end{array}$ \\
\hline $\begin{array}{l}\text { El escolar tiene algún problema emocional o de } \\
\text { comportamiento para el cual necesite ayuda } \\
\text { El escolar está consultando con un profesional de } \\
\text { salud mental }\end{array}$ & $94(32,4)$ \\
\hline & $9(9,6)$ \\
\hline $\begin{array}{l}\text { El escolar tiene algún el } \\
\text { comportamiento para el cual necesite ayuda }\end{array}$ & $96(36,1)$ \\
\hline
\end{tabular}

Tabla 7. Barreras de acceso a la atención de problemas de salud mental, según el apoderado

\begin{tabular}{|lc|}
\hline & n (\%) \\
\hline $\begin{array}{l}\text { No consiguió permiso en el trabajo para buscar } \\
\text { ayuda o consultar a alguien }\end{array}$ & $26(28,9)$ \\
\hline No tenía RUT o FONASA & $29(32,2)$ \\
\hline $\begin{array}{l}\text { No le dio confianza el sistema médico chileno } \\
\text { No conocía como funciona el sistema de salud } \\
\text { público en Chile }\end{array}$ & $44(33,3)$ \\
$\begin{array}{l}\text { Pensó que estos problemas se resolverían solos o } \\
\text { no eran tan graves }\end{array}$ & $51(56,7)$ \\
\hline \begin{tabular}{l} 
Le preocupaba el costo en dinero de la atención \\
\hline
\end{tabular} & $58(64,4)$ \\
\hline
\end{tabular}

Tabla 8. Problemas de salud mental en la población escolar

\begin{tabular}{|lccccc|}
\hline & $\mathbf{n}(\mathbf{\%})$ & $\begin{array}{c}\text { Hombres } \\
\mathbf{n}(\%)\end{array}$ & $\begin{array}{c}\text { Mujeres } \\
\mathbf{n}(\%)\end{array}$ & $\begin{array}{c}\chi^{\mathbf{2}} \\
\mathbf{p}\end{array}$ \\
\hline $\begin{array}{l}\text { Trastornos } \\
\text { del ánimo }\end{array}$ & $43(14,3)$ & $7,6 \%$ & 21,8 & $\begin{array}{c}12,344 \\
\mathrm{(}=0,000\end{array}$ \\
$\begin{array}{l}\text { Trastornos } \\
\text { de ansiedad }\end{array}$ & 17 & $(5,7)$ & $8,9 \%$ & 21,8 & $\begin{array}{c}9,868 \\
\mathrm{p}=0,001\end{array}$ \\
\hline
\end{tabular}

Estos resultados encontrados son compatibles con la literatura internacional que informa tasas inferiores de patología psiquiátrica de la población inmigrante en comparación con la población no inmigrante ${ }^{24}$. Este hecho significa que el impacto del evento inmigración no se traduce en patología psiquiátrica y que los inmigrantes logran adaptarse a sus nuevas condiciones de vida en el país. 
El tipo de trastorno psiquiátrico encontrado -trastornos de ansiedad, y los trastornos del ánimo- es similar al de otros estudios en el que evaluaron la salud mental de población de inmigrantes $^{11,25}$.

La prevalencia de trastornos mentales en la población inmigrante IJ es más alta que la de la población adulta inmigrante consultante al centro de salud primario (29,3\% versus $17,8 \%$ ). Esta diferencia ha sido discutida en la literatura, mostrando que las segundas generaciones de inmigrantes tienen más problemas de salud mental y que tiende a igualarse las prevalencias de la población de los países de acogida ${ }^{2426}$.

La población inmigrante descrita en nuestro estudio es mayoritariamente joven con una percepción de su salud aceptable y que cuando detecta un problema de salud recurre a las instituciones formales del sistema público de salud, en forma importante al consultorio de atención primaria de la comuna de residencia.

En España, se llevó a cabo un estudio acerca de la utilización de servicios de salud por parte de población inmigrantes, comparados con la población residente ${ }^{27}$. Ellos encontraron que la atención médica aumentaba a medida que aumentaban los años de residencia en el país de acogida, lo que atribuyen al conocimiento y adaptación al sistema público de salud adquirido con los años de permanencia en el país.

La atención de salud de la población inmigrante adulta e IJ en Chile presenta barreras relacionadas a la desinformación sobre los derechos de la población inmigrante que tienen los mismos inmigrantes y los funcionarios de salud como lo señala la evidencia internacional ${ }^{14,27}$. Otras barreras de acceso a los servicios de salud encontradas para ambas poblaciones son también coincidentes con la literatura internacional y apuntan a problemas económicos, problemas culturales o de idioma, por ejemplo población latina en Estados Unidos de Norteamérica o africana en Europa ${ }^{16,17,27}$, a diferencia de la migración que se produce actualmente en Chile que proviene de países vecinos que hablan el mismo idioma y tienen una cultura similar.

Es necesario resaltar que la población estudiada percibe como barrera para el acceso a los servicios de salud la carencia de un seguro de salud. Más de $30 \%$ de la población estudiada no tiene FONASA ni ISAPRE probablemente por encontrarse en una situación irregular en el país. Esta variable ha sido reportada como un factor que incide negativamente en la salud de los inmigrantes ${ }^{4}$.

Esto es el primer estudio epidemiológico realizado en Chile sobre la salud mental de la población inmigrante. Las muestras estudiadas son representativas de la población adulta inscrita en el único consultorio de atención primaria que existía en la comuna en esa fecha y de la población IJ escolarizada en la comuna de Independencia. Los instrumentos utilizados permitieron una comparación con estudios de salud mental de la población chilena.

A pesar de estas fortalezas, se trata de un estudio transversal que incluye sólo un municipio. Por ello no es posible generalizar los resultados al resto de los inmigrantes que viven en Chile.

Además aunque los instrumentos han sido ampliamente utilizado en población chilena ${ }^{20}$, pueden haber diferencias transculturales que debiesen ser consideradas en futuras investigaciones ${ }^{28}$.

Si bien es cierto, las cifras de trastornos mentales comunes de la población adulta inmigrante son inferiores a las de la población no inmigrante, las de la población IJ no son despreciables. Es necesario, disminuir las barreras de acceso de esta población a los servicios de salud que cuentan en la actualidad con buenos programas de salud mental e implementar programas de salud escolar que promuevan la salud mental en escolares inmigrantes.

\section{Referencias}

1. Hough RL HA, Soriano FI, Wood P, McCabe K, Yeh M. Mental health services for Latino adolescents with psychiatric disorders. Psychiatr Serv 2002; 53 (12): 1556-62.

2. Kristiansen M. MA, Krasnik A. Health effects of migration. Dan Med Bull 2007; 54 (1): 46-7.

3. Comision Nacional del XVII Censo de Poblacion y VI de Vivienda. CENSO 2002.Instituto Nacional de Estadísticas (INE); 2003.p. 32.

4. Dey AN. LJW. Physical and mental health characteristics of U.S.-andforeign-born adults: UnitedStates, 1998-2003. Hyattsville,: National Center for Health; 2006 Contract No.: Document Number|.

5. Blomstedt Y JS, Sundquist J. Mental health of immigrants from the former Soviet Bloc: a future problem for primary health care in the enlarged European Union? A cross-sectional study. BMC Public Health 2007; 7 (27).

6. Lindert J S-OM, Heinz A, Priebe S. Mental health, health 
care utilisation of migrants in Europe. Eur Psychiatry. 2008; Suppl 1: 14-20.

7. Odegaards O. Emigration and insanity: a study of mental disease among Norwegian born population in Minnesota. Acta Psiquiatr Psicol et Neurol 1932; 7: 1-206.

8. Grant BF SF, Hasin DS, Dawson DA, Chou SP, Anderson K. Immigration and lifetime prevalence of DSM-IV psychiatric disorders among Mexican Americans and non-Hispanic whites in the United States: results from the National Epidemiologic Survey on Alcohol and Related Conditions. Arch Gen Psychiatry 2004; 61 (12): 1226-33.

9. Hough RL, Hazen AL, Soriano FI, Wood P, McCabe K, Yeh M. Mental health services for Latino adolescents with psychiatric disorders. Psychiatr Serv 2002; 53 (12): 1556-62.

10. Levecque K LI, Bracke P. Psychological distress, depression and generalised anxiety in Turkish and Moroccan immigrants in Belgium: a general population study. Soc Psychiatry Psychiatr Epidemiol 2009; 44 (3): 188-97.

11. Wittig U LJ, Merbach M, Brähler E. Mental health of patients from different cultures in Germany. Eur Psychiatry 2008; Suppl 1:28-35.

12. Vollebergh WA tHM, Dekovic M, Oosterwegel A, Pels $\mathrm{T}$, Veenstra R, de Winter A, et al. Mental health in immigrant children in the Netherlands. Soc Psychiatry Psychiatr Epidemiol 2005; 40 (6): 489-96.

13. Aylan SFP, Naske R. Behavioral problems in children of Turkish guest workers in Vienna. Z Kinder Jugendpsychiatr 1993; 21: 226-32.

14. Kirmayer LJ WM, Burgos G, du Fort GG, Lasry JC, Young A. Use of health care services for psychological distress by immigrants in an urban multicultural milieu. Can J Psychiatry 2007; 52 (5): 295-304.

15. Jackson JS NH, Torres M, Martin LA, Williams DR, Baser R. Use of mental health services and subjective satisfaction with treatment among Black Caribbean immigrants: results from the National Survey of American Life. Am J Public Health 2007; 97 (1): 60-7.

16. Chen AW KA. Rate of mental health service utilization by Chinese immigrants in British Columbia. Can J Public Health 2005; 96 (1): 49-51.

17. Sentell TSM, Snowden L. Access to mental health treatment by English language proficiency and race/ ethnicity. J Gen Intern Med 2007; 22 (Suppl 2): 289-93.

18. Araya R WR, Leonard R, Lewis G. Psychiatric morbidity in primary health care in Santiago, Chile. Preliminary findings. Br J Psychiatry 1994; 165 (4): 530-3.

19. Araya R WR, Lewis G. Comparison of two self administered psychiatric questionnaires (GHQ-12 and SRQ-20) in primary care in Chile. Soc Psychiatry Psychiatr Epidemiol 1992; 27 (4): 168-73.

20. Araya RRG, Fritsch R, Acuña J, Lewis G. Common mental disorders in Santiago, Chile: prevalence and socio-demographic correlates. Br J Psychiatry 2001; 178: 228-33.

21. Jellinek M, Murphy J, Robinson J, Feins A, Lamb S, Fenton T. Pediatric Symptom Checklist: screening schoolage children for psychosocial dysfunction. J Pediatr 1988; 112: 201-9.

22. George M, Siraqyan X, Mores R, De La Barra F, Rodríguez J, López C, et al. Adaptación y validación de dos instrumentos de pesquisa de problemas de salud mental en escolares de $1^{\circ}$ básico. Revista de Psicología 1995; 5: 17-25.

23. Vicente PBKR, Saldivia BS, Rioseco SP. Burden of psychiatric diseases in Chile. Rev Med Chile 2007; 135 (12): 1591-9.

24. Breslau J A-GS, Borges G, Kendler KS, Su M, Kessler RC. Risk for psychiatric disorder among immigrants and their US-born descendants: evidence from the National Comorbidity Survey Replication. J Nerv Ment Dis 2007; 195 (3): 189-95.

25. Breslau J A-GS, Kendler KS, Su M, Williams D, Kessler RC. Specifying race-ethnic differences in risk for psychiatric disorder in a USA national sample. Psychol Med 2006; 36 (1): 57-68.

26 Zolkowska K C-GE, McNeil TF. Increased rates of psychosis among immigrants to Sweden: is migration a risk factor for psychosis? Psychol Med 2001; 31 (4): 669-78.

27. Vall-llosera Casanovas L SCC, Sáez Zafra M. Inmigración y salud: necesidades y utilización de los servicios de atención primaria por parte de la población inmigrante en la región sanitaria Girona. Rev Esp Salud Pública 2009; 83 (2): 291-307.

28. García-Campayo J, Sanz C. Salud mental en inmigrantes: el nuevo desafío. Med Clin (Barc) 2002; 118 (5): 187-91. 